\title{
Psychometric properties of the Persian questionnaire health action process approach on the effective communication between nurses and the patient
}

\section{Mojtaba Fattahi Ardakani ${ }^{\mathrm{a}, *}$, Mohammad Ali MorowatiSharifabad ${ }^{\mathrm{b}}$, Mohammad Amin Bahrami ${ }^{\mathrm{c}}$, Hossein Fallahzadeh ${ }^{\mathrm{d}}$}

${ }^{a}$ Health Education \& Health Promotion, Healthcare Services Department, Shahid Sadoughi University of Medical Sciences, Yazd, Iran

${ }^{\mathrm{b}}$ Health Education Department, Shahid Sadoughi University of Medical Sciences, Yazd, Iran

${ }^{\mathrm{c}}$ Healthcare Management Department, School of Management and Medical Informatics, Shiraz University of Medical Sciences, Shiraz, Iran

${ }^{\mathrm{d}}$ Biostatistics and Epidemiology Department, Shahid Sadoughi University of Medical Sciences, Yazd, Iran

\section{A R T I C L E I N F O}

\section{Keywords:}

Psychometric properties

Scale validation

Interprofessional communication

Questionnaire

Nurse-patient communication

\begin{abstract}
A B S T R A C T
Introduction: Patient communication is one of the key elements of nursing care that in many cases this relationship is not effectively established. Effective communication with the patient can lead to patient satisfaction and improve health outcomes. Therefore, the aim of this study was to determine the psychometric properties of the questionnaire Based on the Health Action Process approach (HAPA) in the field of effective communication between the nurse and the patient.

Methods: This cross-sectional study was carried out in 2018 in nurses of Yazd hospitals in the center of Iran. We selected a total of 300 nurses selected randomly from the four hospitals (75 nurses from each hospital). The content and form of the questionnaire were approved by the panel of experts. Subsequent 289 nurses complete the questionnaire. To evaluate validity, differential and structural model, software PLS V 3 was used. Results: Content validity was acceptable $(\mathrm{CVR}=0.79, \mathrm{CVI}=0.80)$. The results of the scales Structural approvals (CI $>0.7$, AVE $>0.5$ and (VIF = 1. 36) were confirmed. Fernel Larker's score also confirmed differential correlation. The significance problem was greater than 1.96, indicating fit for model estimation.

Conclusion: The findings of this study confirm the reliability and validity of HAPA structures in the context of effective communication between the nurse and the patient.
\end{abstract}

\section{Introduction}

Effective nurse-patient communication is one of the main components to efficient care. ${ }^{1}$ Nursing staff are required to be good at communication. They will need to ensure that patients have all necessary information about their condition and identify and exhibit their patients Concerns. ${ }^{2}$ The essential prerequisites for successful communications between healthcarers and their patients are that the amount details given is adequate, which it is understood, believed, remembered and hopefully, put to work. ${ }^{3}$ Effective communication leads to identifying problems, concerns, and diagnosis. Patient satisfaction, compliance with the care plan, and self-management improvement of the disease are achieved through more effective communication. In case of establishing an effective and effective communication, reducing risks will have a positive effect on the patients, including improving vital health, reducing pain and anxiety and reducing hospitalization time. ${ }^{3}$
Studies show that despite the fact that communication skills are taught to nurses in educational settings, nurses do not use these skills in a permanent manner in the clinic. The reason for this conflict can be the existence of a gap between competence and merit (what a person can or can do) and performance (what the person really does) that the patient's dissatisfaction points to this point. ${ }^{4,5}$ Several studies have examined the effective factors in the lack of effective communication between nurses. Some of these factors include nurse's importance to communication, nurse's knowledge of the disease process, nurse's willingness to communicate, nurse's confidence, work crowding, communication skills and communication, proper implementation of communication skills, planned communication, Nurse's emotions, patient's emotions and companionship, lack of understanding of the benefits of patient communication and low self-efficacy. ${ }^{6-9}$ Also, the perception of the personnel of their skills can affect the quality of intervention in the patient and accompany the patient's dissatisfaction with. ${ }^{10-12}$ To improve

\footnotetext{
* Corresponding author.

E-mail addresses: mojtabafattahiardakani@gmail.com (M.F. Ardakani), morowatisharif@yahoo.com, mjfattahi57@gmail.com (M.A. MorowatiSharifabad), aminbahrami1359@gmail.com (M.A. Bahrami), hofaab@yahoo.com (H. Fallahzadeh).
} 
nurses' communication skills, the use of a theory-based intervention can help in the implementation, targeting, monitoring of intervention and achievement of specified goals. ${ }^{11,12}$ In previous studies, the barriers to communication were mentioned. ${ }^{6,7,13}$ The constructs of the Health Action process approach(HAPA) can be effective in reducing barriers. Self -efficacy, perceived risk, outcome expectancies and planning are of important constructs in the HAPA. These constructs of HAPA considered in studies. But they have not examined the obstacles and mechanisms of promoting communication skills systematically. Thus this Approach can be effective for enhancing skills communication in nurses. In most cognitive-social theories, it is assumed that the individual's intention to change the best predictor of direct change is real. But people often do not behave according to their intentions. This is the difference between the intention and the reason for a number of reasons. Unpredictable barriers can emerge, or people may be tempted to surrender. These factors may play a role in interacting with the intent to act. Some of these factors have been identified after intentional selfefficacy and strategic planning. One of the social cognitive models that has paid special attention to these issues is the approach to the health process. Unlike other cassette models that put people in a group, they are based on how they are behaved. Separate and identify the intervention appropriately based on what stage they are. ${ }^{14}$ Changing the behavior of health refers to the processes of deliberative, decisionmaking and practical behavior. This change involves avoiding behaviors that compromise health or the adoption and maintenance of health-enhancing behaviors. This approach involves factors that arise after intention to interfere with the intention to engage in action. In this approach, there is a pre-intentioned motivation process (such as perceived risk) that leads to motivation for behavior change and voluntary process there is a distinction between the motives that leads to a change in real behavior. The motivation phase is a process in which a person intends to take action or change a high-risk behavior. In a voluntary phase, the individual decides to conduct new behaviors, and includes three steps: start, maintenance, and recovery. ${ }^{14}$ (see Fig. 1)

In the motivation stage, the person wants to do something. In this phase, perceived danger is considered as an early advance, which alone is not enough to create intent on the person. ${ }^{15,16}$ In the motivation stage, when the person examines the benefits and disadvantages of certain behavioral outcomes, the expectations of the positive results are important. In addition, a person needs to have the ability to perform an action desirable, which is referred to as perceived self-efficacy. Perceived self-efficacy works in concert with expectations, with positive results, which both co-operate in the formation of intent. Once a person has a tendency to a particular hygiene behavior, the intention is deformed to the detailed instructions. Therefore, the stage after the intention is to decompose planning action and recovery self-efficacy.When a behavior is approved, the intention is to turn into action. For example, if a person wants to lose weight, they have to design how to do it, such as what to buy, when and how much to eat, when and where to exercise. Therefore, a comprehensive intention can be formed by a set of affiliates and action planning. The results of Barbara et al. confirmed the dimensions of the HAPA that can contribute to the promotion of health food preparation. ${ }^{15,17}$ Although several studies have addressed the barriers for effective nurse communication with the patient, the authors' searches have not addressed changes in behavior theory to the difference between those who have effective communication with the patient and those who do not intend to promote effective communication. The correct instrument is the proper measurements of the variables in question. While the inappropriate tool leads to the collection of non-relevant information and the non-scientific interpretation of the findings of the research, the questionnaire design should be psychometric. ${ }^{18}$ Considering the lack of Persian version of the persistent and persistent health process approach to explain the effective communication with the patient by nurses and since the difference in the society in question can affect the validity and reliability of the tool, this study aims to psychometric assessment of the HAPA in the field of effective communication between nurse and patient in selected hospitals in Yazd, Iran.

\section{Method}

This cross-sectional study was carried out in 2018 selected hospitals in Yazd. Questionnaire data were used in previous studies of the relationship between the nurse and the patient, which was utilized in this approach. ${ }^{4,6,10}$ The questionnaire was designed based on the HAPA guideline. ${ }^{15}$ The questionnaire was used based on previous studies in

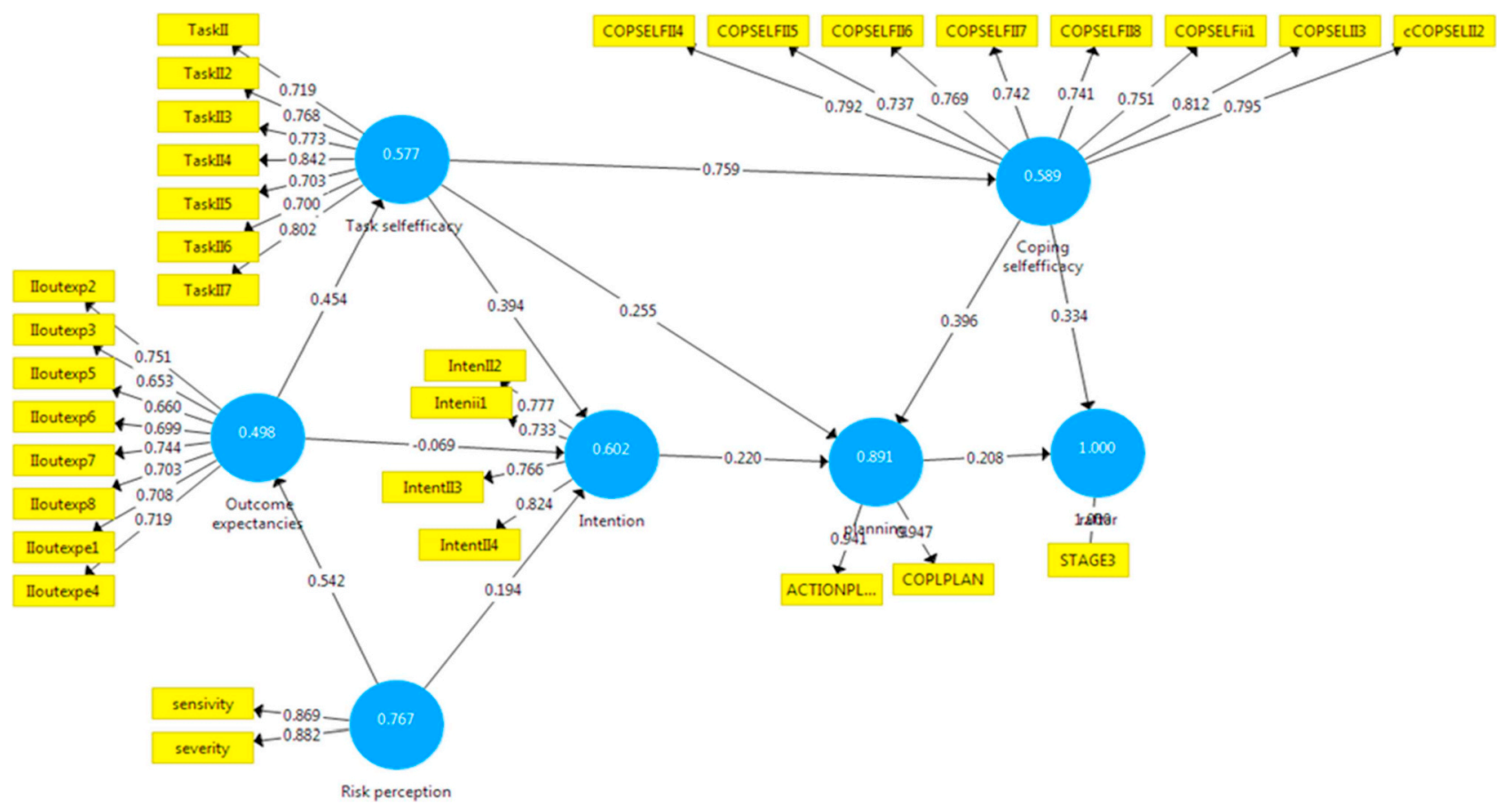

Fig. 1. Path regression model. 
this field. In order to confirm the scientific content, the questionnaire was investigated by 8 panel of experts. Their comments were made in this regard. CVR andCVI were confirmed. The initial questionnaire consisted of 8 scales as follows:

Perceived susceptibility ( 9 items), perceived severity ( 9 items), outcome expectation ( 8 items), Task self-efficacy ( 7 items), intent (4 items), Coping self-efficacy (8 items), behavioral planning (5 items), and Coping planning (7 items) and behavior (one question).

The criterion of judgment in the questionnaire of the Likert spectrum is the question of "completely correct" to "completely false" for self-efficacy measures, self-efficacy, behavioral planning, and planningassertiveness. For the perceived susceptibility scale of the Likert spectrum, there are 5 questions of "very high probability" to "not at all probable", the Likert spectrum has 5 questions from "very large" to "at all" for the intensity scale, and for the "intent" Too much used.

This scale, according to Leedy and Ormrod's guidance, ${ }^{19}$ facilitated the accountability process because of the broader range of responses and the inclusion of "no opinion." Based on the approach of the health process, people are at different stages of the behavior. Therefore, the question is how nurses will be effective at communicating with each other. In this question, the nurse should choose one of the options: a) I do not have an effective relationship. And I have no decision to improve communication skills. B: I have no effective communication with the patient, but I am very eager to learn and have effective communication. I have effective communication with the patient, but it is difficult for me to: I have a good relationship with the patient easily.

To determine the formal validity in the qualitative section, 20 nurses were included in the questionnaire. In the context of the level of difficulty of the concepts, the degree of fitness and communication, and the importance of the items and the elimination of inappropriate items, a quantitative effect method was applied which had a quantitative score Over 1.5 was considered acceptable. The subjects were not included in the statistical population. Minor changes were made to clarify the questions.

In order to determine the content validity in the quantitative section, it was also used by the experts. First, the content validity ratio was calculated and then the content validity index was calculated. The credit validity indicates that the scale was successful in achieving certain goals. However, the reliability coefficients are usually greater than the credit coefficients, but credibility is always more important. ${ }^{19}$ Content validity was computed using three levels: "Item not necessary, item is useful but not necessary, item is necessary."

After calculating the proportion by referring to the Brand 2008 table, ${ }^{20}$ if the number calculated by the formula for each item was larger than the number given in the Lawshe table, that item was considered essential and important for further analysis. Became The Content Validity Index specifies whether the tool items are best measured by the health process approach structures, and that the content validity index is more than 0.79 . The three criteria are "simplicity and fluidity" Being, Relevant and clear or Transparent "was used with the use of the 4-part Likert Spectrum for each item.

Without a reliable tool, the results of the research cannot be sufficiently reliable. If the research is repeated, the results can have a significant difference with the first stage. However, the reliability is not a sufficient condition to rely on the results of a measurement tool, but a necessary condition. ${ }^{21}$ The internal consistency measurement was performed using the Cronbach's alpha coefficient and values equal to more than 0.65 were considered acceptable. Cronbach's alpha coefficient in various sciences, especially the human and medical sciences, to measure the reliability of different attitudes measurement tools or educational prototypes and knowledge measurement are abundant. After the Cronbach alpha coefficient design, B Annual average of 131 times a scientific article written by Lee J. Cronbach's been used in this regard. ${ }^{22}$ Thirty subjects were selected in a preliminary study to examine this test. They were selected from a hospital other than the study. After collecting, Cronbach's alpha coefficient was calculated using SPSS software for the questionnaire. The Cronbach's alpha coefficient was 0.87. The calculation of Cronbach's alpha at this stage showed that the designed questions had a satisfactory internal reliability and therefore, at the initial stage of our implementation, no questions were eliminated due to lack of internal reliability.

\section{Sample size and sampling}

In this cross-sectional study, nurses were included from Shahid Sadoughi and Seyed al-Shohada hospitals in Yazd, Imam Jafar Sadeq hospital in Meybod and Ziaei hospital in Ardakan. Nurses who have been working in internal, general surgery, cardiology, pediatric and gynecology departments for at least one year were enrolled in the study. $\mathrm{G}$ power 3 analysis software ${ }^{23}$ was used to calculate the required sample size. Regarding the significant level of 0.05 , average effect size of 0.3 , number of variables ${ }^{7}$ and analysis power of 0.99 , the sample size was estimated 270 . Taking into account the response rate, we selected a total of 300 nurses selected randomly from the four hospitals (75 nurses from each hospital). The selected nurses were asked to answer the questionnaires completely. Incomplete questionnaires were excluded from the analysis. Finally, the data drawn from 289 questionnaires were analyzed. Data collection were done from February 2018 to March 2018. Nurses who were not just a few months after they started or retired at the end of the year were not included in the study. From 306 people, 289 returned the questionnaire and 10 answered incomplete questions. The response rate was $91 \%$. The number of subjects for psychometric studies was a desirable standard for sample size. In the study of the structural model, perceived risk constructs were used that consisted of two perceived sensitivity and perceived severity structures. Also, in the model of two planning structures Behavior and planning were subdivided into planning.

\section{Data analysis}

Also, in this research, the patterning of the structural modalities and the partial least squares method (PARTIAL LEAST SQUERS) were used for hypotheses and pattern matching. PLS programming is used in various sciences, including human resources and health. ${ }^{24,25}$ The PLS method is tested with two patterns. The first model is external, or a measurement model that includes the questions and the variable, and the second model is an internal or structural pattern that shows the variables and relations between them. ${ }^{26}$ In order to verify the reliability of the structure, three components of composite reliability (CR), extracted mean variance (AVE) and factor loading (FAR) were used. The stability condition of the structure is that the composite reliability value (CR) is greater than 0.7 and the size the average extracted variance (AVE) is greater than $0.5 .{ }^{27}$ To investigate the validity of the construct, two types of convergent and divergent validity were investigated. The meaning of convergent validity is whether the units measure precisely what we mean. Divergent validity also checks that the terms for each specific variable are not measured by the extent to which other variables are present. The condition for constructing the convergent validity is that the composite stability dimension for each construct is larger than the average extracted variance (CR > AVE). To investigate the divergent validity, the method used by Fornell and Larker was used. They argue that divergent validity is at an acceptable level that the AVE for each construct has a distribution of variance between that construct and other structures, ie, the square of the correlation coefficients between the structures in the pattern is more. The model has a reasonable divergent validity if the numbers in the original diameter are larger than their lower size. ${ }^{27}$ The state of forecasting variance for independent predictor variables also shows that each of the independent variables has its own unique role and there is no significant interference in the formulation of the explained variance of the model. In the field of PLS-SEM VIFs of 5 or more represent potential synchronization problems, respectively. These levels indicate that $80 \%$ of the variance of a 
reagent by other residual reagents is related to the same structure. ${ }^{27}$ Thus, the average inversion factor of less than 5 indicates a moderate fit and less than 3.30 indicates good fit. ${ }^{28}$ Structural model fit: Structural model section, unlike measuring models, does not have any obvious variables, and only hidden variables are investigated along with the relationships between them. Also, for parametric meaning, the parameters were applied to the nonparametric Bootstrap procedure (with 130 replications). In Bootstrap, a large number of submenus are drawn from the original sample by placement method. These methods are used to estimate the PLS path model. In this case the method of $\mathrm{z}$ coefficient coefficients (values of t-values) is obtained. To fit the model, these coefficients should be more than 1.96 so that they can be confirmed in 95\% confidence intervals. ${ }^{29}$ For general fit, the Goodness of Fit (GOF) criterion was used using pls output data. The general fitness criterion (GOF) can be obtained by calculating the mean geometric mean of the subscription and $\mathrm{R}^{\wedge} 2$. According to Tenon House et al., the GOF index in the PLS model is a practical solution for examining the general fit of the model and, Fit in covariance based methods and can be used to check the validity or quality of the PLS model in general. This index acts as well as the fitting indices of the LaserLeam model and is between zero and one, and the values of 0.10.0.25 and 0.36, respectively, show small, medium and large effect sizes. ${ }^{25}$

\section{Results}

Most married participants $(89.1 \%)$ were female (88.3\%). The mean age of nurses was $33.5 \pm 7.05$ and more in the age range of 22-52 years. In terms of education, most of them had a bachelor's degree in nursing $(81 \%)$. Their average working experience was $10.26 \pm 7.82$.

The qualitative validity of the qualitative section was based on the reflections received from the individuals, minor and necessary changes were made for complete clarity. The effect of all items was above 1.5. In the qualitative content validity sense, some experts had some modest comments that the questionnaire was applied. Therefore, the content validity qualitative results were confirmed. In the next step, the results of quantitative content validity were investigated. The content validity ratio was also valid except for 4 cases, for all above 0.79 , and its values after removal of 4 the case for each of the structures is presented in Table 1. The final questionnaire consists of 9 scales as follows: Iodine:

Perceived sensitivity (7 items), perceived severity (7 items), outcome expectancies (8 items), Task self-efficacy (7 items), intention (4 items), Coping self-efficacy ( 8 items), Action planning (5 items), and Coping planning (7 items).

Table 1 shows the reliability of the constructs and the content validity index. In all constructs, the Mean Cronbach's alpha was higher than 0.60 .

The mean of the variance extracted in most of the structures was higher than 0.5. Also, the composite reliability coefficient was higher than 0.7 and this coefficient was higher than the AVE in the same structure, which shows the convergent validity of the structures.

As shown in Table 2, the AVE value of the present research variables, which are located in the main matrix of the matrix, is greater than the correlation between them located in the lower and the righthand side of the main diameter. Therefore, it can be stated that in this research, structures in the model interact more with their indices than with other structures. That is, the divergent validity of the model is appropriate.

Factor load is one of the main criteria for checking factor verification. In this study, the yield factor for all items was more than 0.5 , indicating confirmation of the items (Table 3). Considering the coefficients $t$ and their significant level (Table 4), the perceived risk variable at the $95 \%$ confidence level affects outcome variables, behavioral intention, and task self-efficacy. Also, the results show that behavioral expectation variable at the level The error of less than $5 \%$ has no significant effect on the behavioral intention variable. The self-efficacy and reliability variable at the level of $95 \%$ confidence and $5 \%$ error

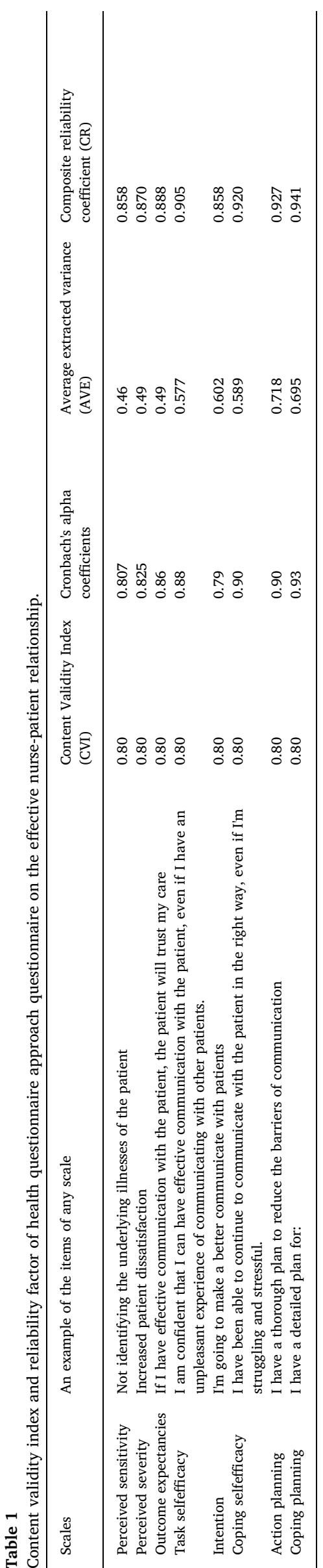


Table 2

The divergent validity of the matrix according to Fornell and Larker's method.

\begin{tabular}{|c|c|c|c|c|c|c|c|c|c|}
\hline Variables & $\begin{array}{l}\text { Action } \\
\text { planning }\end{array}$ & $\begin{array}{l}\text { Coping } \\
\text { planning }\end{array}$ & $\begin{array}{l}\text { Outcome } \\
\text { expectancies }\end{array}$ & Behavior & $\begin{array}{l}\text { Coping self } \\
\text { efficacy }\end{array}$ & Intention & $\begin{array}{l}\text { Perceived } \\
\text { sensitivity }\end{array}$ & $\begin{array}{l}\text { Perceived } \\
\text { severity }\end{array}$ & $\begin{array}{l}\text { Action self } \\
\text { efficacy }\end{array}$ \\
\hline Action planning & 0.847 & & & & & & & & \\
\hline Coping planning & 0.783 & 0.834 & & & & & & & \\
\hline Outcome expectancies & 0.241 & 0.311 & 0.705 & & & & & & \\
\hline Behavior & 0.405 & 0.419 & 0.218 & $\begin{array}{l}\text { Single item } \\
\text { criterion }\end{array}$ & & & & & \\
\hline Coping self efficacy & 0.628 & 0.666 & 0.384 & 0.476 & 0.768 & & & & \\
\hline Intention & 0.466 & 0.475 & 0.215 & 0.347 & 0.437 & 0.776 & & & \\
\hline Perceived sensitivity & 0.203 & 0.252 & 0.429 & 0.147 & 0.256 & 0.252 & 0.682 & & \\
\hline Perceived severity & 0.182 & 0.193 & 0.510 & 0.139 & 0.188 & 0.172 & 0.540 & 0.700 & \\
\hline Action self efficacy & 0.582 & 0.636 & 0.455 & 0.393 & 0.759 & 0.414 & 0.230 & 0.197 & 0.760 \\
\hline
\end{tabular}

margin had the greatest impact on self-efficacy (0.762). The mean of the AVIF factor in this study was 1.34, which indicates a lack of linearity in the structural model.

The fitting of the general model was calculated using the formula $\mathrm{GOF}=$ VAVG (Comunalitie) $* \mathrm{AVGR}^{\wedge} 2$

$\sqrt[2]{0.92 * 0.53}=0.70$

Given the obtained result, it was fitted with a structural fit.

\section{Discuss}

Effective communication with the patient is one of the pillars for improving patient care. Effective communication will improve the outcome of the patient's health and satisfaction. Therefore, understanding this issue and enhancing the communication skills of the nurse with the patient is one of the important points to be considered. Studies show that the nurse has an effective communication with the patient. The health action process proposes the difference between nurses in terms of effective communication with the patient. A review of past studies has shown that the health process approach to explaining the differences between nurses in effective communication has not been used. Therefore, this study was conducted with the purpose of psychometric evaluation of a suitable tool in this regard. The results of the content validity index in this study were higher than 0.79 which indicates the validity of the questionnaire. The reliability of a tool is indicative of the accuracy or accuracy of the measurement. Because of nonparametric data, the PLS method was used to examine the model. ${ }^{30}$ The statistical validation strategy offered herein follows most of the advice of good practice for score affirmation. ${ }^{31}$ The results of this study showed that all of the constructs of the questionnaire had a high Cronbach's alpha coefficient, which is a strong confirmation of the questionnaire. The result was also found in Renner, Pierre, and Martinez, Lhakhang studies, which reported the Cronbach's alpha coefficient between 0.70 and $0.93{ }^{29-32}$ The results of the major input, AVE and CR are also confirmatory of the questionnaire Persian version. The total score was more than 0.5. A convergent and divergent version of the confirmation of this questionnaire for its use. The results provided initial proof| for the validity of the HAPA-based measures. Nevertheless there may be a slight sample bias considering the nature of interest of nursese about communication, within that collected data may well not be representative for the focus on group. Also, external quality may be restricted.

Confirmation of the questionnaire means that the scale of the health process approach correctly measures the scale of the questionnaire. The results of Farnell Larker's table indicate the appropriateness of the divergence of the model. This indicates the differentiation of a structure with other structures, and what is in This structure is obtained not in other model structures. ${ }^{29}$ Structural equation modeling showed that perceived risk to $95 \%$ confidence level had an effect on outcome expectatancies and behavioral intention. In HAPA perceived risk as one of the variables influencing people to promote or not perform health behaviors Health behaviors. ${ }^{19}$ This result is consistent with other studies such as perceived risk of smoking cessation. ${ }^{32}$

The result of this study is a lack of correlation between outcome expectation and behavioral intention. This result is also seen in other studies ${ }^{33,34}$ and can be due to the average score of nurses. Nurses who have a high score of empathy with the patient they wanted to have less empathy. ${ }^{35}$ Also, the expectation of a high outcome could show the impact of self-efficacy, which in this study also had a high correlation with self-efficacy. The self-efficacy of the behaviors, behavioral intention, had a positive impact on the program they were aligned with the results of other studies on the use of HAPA. ${ }^{15,36-39}$

The average of the variation curvature factor (AVIF) in this study indicates that we can use the results of external weights in the developmental models. ${ }^{29}$ The HAPA structures were able to predict effective communication. The results confirm the use of this Persian version in the prediction of communication skills using HAPA constructs and can be used by identifying the need for persuasive effective communication by using the pre-intervention questionnaire. Also, after persuading them to communicate effectively using Action Planning, Coping Planning, and Auto-Efficiency Conducted appropriate interventions to promote effective communication. In many interventions, lack of a pattern

Table 3

Results of factor analysis of health questionnaire health inventory questionnaire on the effective relationship between nurse and patient.

\begin{tabular}{|c|c|c|c|c|c|c|c|c|c|c|c|c|c|c|c|}
\hline \multicolumn{2}{|c|}{ Coping planning } & \multicolumn{2}{|c|}{ Action planning } & \multicolumn{2}{|c|}{ Coping self efficacy } & \multicolumn{2}{|l|}{ Intention } & \multicolumn{2}{|c|}{ Task selfe fficacy } & \multicolumn{2}{|c|}{ Outcome expectancies } & \multicolumn{2}{|c|}{ Perceived severity } & \multicolumn{2}{|c|}{ Perceived sensitivity } \\
\hline $\begin{array}{l}\text { Load } \\
\text { capacity }\end{array}$ & Items & $\begin{array}{l}\text { Load } \\
\text { capacity }\end{array}$ & Items & $\begin{array}{l}\text { Load } \\
\text { capacity }\end{array}$ & Items & $\begin{array}{l}\text { Load } \\
\text { capacity }\end{array}$ & Items & $\begin{array}{l}\text { Load } \\
\text { capacity }\end{array}$ & Items & $\begin{array}{l}\text { Load } \\
\text { capacity }\end{array}$ & Items & $\begin{array}{l}\text { Load } \\
\text { capacity }\end{array}$ & Items & $\begin{array}{l}\text { Load } \\
\text { capacity }\end{array}$ & Items \\
\hline 0.79 & 1 & 0.83 & 1 & 0.55 & 1 & 0.92 & 1 & 0.53 & 1 & 0.69 & 1 & 0.59 & 1 & 0.58 & 1 \\
\hline 0.77 & 2 & 0.82 & 2 & 0.67 & 2 & 0.93 & 2 & 0.70 & 2 & 0.74 & 2 & 0.70 & 2 & 0.73 & 2 \\
\hline 0.76 & 3 & 0.84 & 3 & 0.75 & 3 & 0.72 & 3 & 0.65 & 3 & 0.64 & 3 & 0.75 & 3 & 0.67 & 3 \\
\hline 0.77 & 4 & 0.90 & 4 & 0.72 & 4 & 0.82 & 4 & 0.72 & 4 & 0.71 & 4 & 0.75 & 4 & 0.72 & 4 \\
\hline 0.84 & 5 & 0.88 & 5 & 0.74 & 5 & & & 0.63 & 5 & 0.67 & 5 & 0.67 & 5 & 0.64 & 5 \\
\hline 0.86 & 6 & & & 0.76 & 6 & & & 0.71 & 6 & 0.68 & 6 & 0.73 & 6 & 0.73 & 6 \\
\hline \multirow[t]{2}{*}{0.77} & 7 & & & 0.72 & 7 & & & 0.96 & 7 & 0.73 & 7 & 0.68 & 7 & 0.66 & 7 \\
\hline & 8 & & & 0.73 & 8 & & & & & 0.69 & 8 & & & & \\
\hline
\end{tabular}


Table 4

Correlation matrix between variables.

\begin{tabular}{|c|c|c|c|c|}
\hline Effective constructs & Path coefficient & statisticts & standard error & Significance level \\
\hline Risk perception $\rightarrow$ Intention & 0.19 & 2.888 & 0.066 & 0.004 \\
\hline Risk perception $\rightarrow$ Outcome expectancies & 0.542 & 13.039 & 0.042 & $<0 / 001$ \\
\hline Coping self efficacy $\rightarrow$ Effective communication & 0.396 & 4.724 & 0.084 & $<0 / 001$ \\
\hline Coping self efficacy $\rightarrow$ Planning & 0.334 & 4.308 & 0.078 & $<0 / 001$ \\
\hline Intention $\rightarrow$ Planning & 0.22 & 3.80 & 0.05 & $<0 / 001$ \\
\hline Outcome expectancies $\rightarrow$ Intention & -0.06 & 0.89 & 0.07 & 0.37 \\
\hline Outcome expectancies $\rightarrow$ Task self efficacy & 0.45 & 8.69 & 0.05 & $>0 / 001$ \\
\hline Planning $\rightarrow$ Effective communication & 0.20 & 2.616 & 0.08 & $<0 / 001$ \\
\hline Task self efficacy $\rightarrow$ Coping self efficacy & 0.76 & 23.99 & 0.032 & $<0 / 001$ \\
\hline Task self efficacy $\rightarrow$ Intention & 0.40 & 6.090 & 0.06 & $<0 / 001$ \\
\hline Task self efficacy $\rightarrow$ Planning & 0.25 & 3.352 & 0.07 & $<0 / 001$ \\
\hline
\end{tabular}

or approach would lead to non-achievement of goals. Also, to achieve Objectives, identifying a strategy using a template can be of help. Therefore, to promote the effective use of this approach can be useful. ${ }^{11,14,40}$ The use of a model or theory has been used in many studies as Parle et al. ${ }^{24}$ Used the development of communication skills from HAPA structures.

\section{Conclusion}

In general, considering the reliability of the questionnaire and the high reliability and fit of the structural model of the Persian version, it can be said that the process of health practice in promoting communication skills is a good tool and can be used in similar research. From the limitations of this the study of the use of an item in the study of effective communication behavior should be considered in other studies. Also, the limitations of this study are the use of sampling in an easy way, but with the use of several hospitals in this study, a representative of the nursing group. Also, the culture of nurses in Yazd province the cause of the limitations of this study.

\section{Ethics approval and consent to participate}

The participants were assured that their information would be kept confidential, and then they completed questionnaires. Moreover, the participation in the study was voluntary. The study protocol was approved by the Ethics Committee the shahid sadoughi University of Medical Sciences, Yazd, Iran (code: IRSSU.SPH.REC.1395.76).

\section{Acknowledgments}

we really appreciate of all those who collaborated with the researchers in this study, including the directors and nurses of Shahid Sadoughi Hospital in Yazd, Seyed al-Shohada, Imam Jafar Sadegh in Meybod and Ziaei in the city of Ardakan, I would like to express my sincere appreciation. This article is part of Ph.D thesis on health education and promotion at Shahid Sadoughi University of Medical Sciences in Yazd,Iran.

\section{References}

1. Finke EH, Light J, Kitko L. A systematic review of the effectiveness of nurse communication with patients with complex communication needs with a focus on the use of augmentative and alternative communication. $J$ Clin Nurs. 2008:17(16):2102-2115.

2. Moulton C-a, Tabak D, Kneebone R, Nestel D, MacRae H, LeBlanc VR. Teaching communication skills using the integrated procedural performance instrument (IPPI): a randomized controlled trial. Am J Surg. 2009;197(1):113-118.

3. Bowles N, Mackintosh C, Torn A. Nurses' communication skills: an evaluation of the impact of solution-focused communication training. J Adv Nurs. 2001;36(3):347-354.

4. Liu J-E, Mok E, Wong T, Xue L, Xu B. Evaluation of an integrated communication skills training program for nurses in cancer care in beijing, China. Nurs Res. 2007;56(3):202-209 PubMed PMID: 00006199-200705000-00008.

5. Johansson P, Oléni M, Fridlund B. Patient satisfaction with nursing care in the context of health care: a literature study. Scand J Caring Sci. 2002;16(4):337-344

6. Sheldon LK, Barrett R, Ellington L. Difficult communication in nursing. J Nurs Scholarsh. 2006:38(2):141-147.

7. Park E-k, Song M. Communication barriers perceived by older patients and nurses. Int J Nurs Stud. 2005;42(2):159-166 2005/02/01/

8. Hemsley B, Balandin S, Worrall L. Nursing the patient with complex communication needs: time as a barrier and a facilitator to successful communication in hospital. $J$ Adv Nurs. 2012;68(1):116-126.

9. Anoosheh M, Zarkhah S, Faghihzadeh S, Vaismoradi M. Nurse-patient communication barriers in Iranian nursing. Int Nurs Rev. 2009;56(2):243-249.

10. Fleischer S, Berg A, Zimmermann M, Wüste K, Behrens J. Nurse-patient interaction and communication: a systematic literature review. J Public Health. 2009 October 01;17(5):339-353.

11. Parle M, Maguire P, Heaven C. The development of a training model to improve health professionals' skills, self-efficacy and outcome expectancies when communicating with cancer patients. Soc Sci Med. 1997;44(2):231-240.

12. Oates J, Weston WW, Jordan J. The impact of patient-centered care on outcomes. Fam Pract. 2000;49:796-804.

13. Fallowfield L, Jenkins V. Effective communication skills are the key to good cancer care. Eur J Cancer. 1999;35(11):1592-1597.

14. Glanz K, Bishop DB. The role of behavioral science theory in development and implementation of public health interventions. Annu Rev Public Health. 2010;31(1):399-418 PubMed PMID: 20070207.

15. Schwarzer R. Modeling health behavior change: how to predict and modify the adoption and maintenance of health behaviors. Appl Psychol. 2008;57(1):1-29.

16. Schwarzer R, Sniehotta FF, Lippke S, et al. On the Assessment and Analysis of Variables in the Health Action Process Approach: Conducting an Investigation. Berlin: Freie Universeitat Berlin; 2003

17. Sutton S. How does the health action process approach (HAPA) bridge the intention-behavior gap? An examination of the model's causal structure. Appl Psychol 2008;57(1):66-74.

18. Nunnally JC, Bernstein IH. Psychometric Theory. New York: McGraw-Hill; 1994

19. Leedy PD, Ormrod JE. Practical Research: Planning and Design. 10th. New Jersey: Pearson Education Limited; 2013

20. Teti DM, Gelfand DM. Behavioral competence among mothers of infants in the first year: the mediational role of maternal self-efficacy. Child Dev. 1991;62(5):918-929.

21. Ghasemi V. Structural Models in Social Science Research. Isfahan: Jahad Daneshghahi Press; 2010 [In Persian].

22. Cronbach LJ, Shavelson RJ. My current thoughts on coefficient alpha and successor procedures. Educ Psychol Meas. 2004;64(3):391-418.

23. Kline RB. Principles and Practice of Structural Equation Modeling. 3 rd ed. New York, NY: The Guilford Press; 2011.

24. Wu WW. Linking Bayesian networks and PLS path modeling for causal analysis Expert Syst Appl. 2010;37(1):134-139.

25. Tenenhaus M, Vinzi VE, Chatelin Y-M, Lauro C. PLS path modeling. Comput Stat Data Anal. 2005;48(1):159-205.

26. Hulland J. Use of partial least squares (PLS) in strategic management research: a review of four recent studies. Strat Manag J. 1999:195-204.

27. Fornell C, Larcker DF. Evaluating structural equation models with unobservable variables and measurement error. J Mark Res. 1981:39-50.

28. Kock N. WarpPLS 5.0 User Manual. 2015. Laredo, TX: ScriptWarp Systems; 2015.

29. Hair JF, Hult GTM, Ringle CM, Sarstedt M. A Primer on Partial Least Squares Structural Equation Modelin(Translate from Adel Azar and Rasoul Gholamzadeh). vol. 1. Tehran: Negahedanesh; 2016359 pp.

30. Wold S. Soft Modeling: The Basic Design and Some Extensions, Systems under Indirect Observations. Causality Structure Prediction Ed KG Joreskog H Wold. Amsterdam: North Holland Publishing Company; 1982

31. Coste J, Fermanian J, Venot A. Methodological and statistical problems in the construction of composite measurement scales: a survey of six medical and epidemiological journals. Stat Med. 1995;14(4):331-345.

32. Williams RJ, Herzog TA, Simmons VN. Risk perception and motivation to quit smoking: a partial test of the Health Action Process Approach. Addict Behav. 2011;36(7):789-791 2011/07/01/.

33. Martinez-Brockman JL, Shebl FM, Harari N, Pérez-Escamilla R. An assessment of the social cognitive predictors of exclusive breastfeeding behavior using the Health Action Process Approach. Soc Sci Med. 2017;182:106-116. 
34. Renner B, Kwon S, Yang B-H, et al. Social-cognitive predictors of dietary behaviors in South Korean men and women. Int J Behav Med. 2008 March 01;15(1):4-13.

35. Pollak KI, Arnold RM, Jeffreys AS, et al. Oncologist communication about emotion during visits with patients with advanced cancer. J Clin Oncol. 2007;25(36):5748-5752.

36. Renner B, Schwarzer R. Social-cognitive factors in health behavior change. Social psychological foundations of health and illness. 2003:169-196.

37. Lippke S, Ziegelmann JP, Schwarzer R. Stage-specific adoption and maintenance of physical activity: testing a three-stage model. Psychol Sport Exerc. 2005;6(5):585-603 2005/09/01/.
38. Norouzi S, Moghaddam MHB, Morowatisharifabad MA, Norouzi A, Jafari AR, Fallahzadeh H. Examining social-cognitive predictors of parenting skills among mothers with preschool and early elementary school-aged children. $J$ Educ Health Promot. 2015 12/30;4:96 PubMed PMID: PMC4946277.

39. Wiedemann AU, Lippke S, Reuter T, Schüz B, Ziegelmann JP, Schwarzer R. Prediction of stage transitions in fruit and vegetable intake. Health Educ Res. 2009;24(4):596-607.

40. Stajkovic AD, Luthans F. Self-efficacy and work-related performance: a meta-analysis. Psychol Bull. 1998;124(2):240. 